\title{
A Study of the Oil Loss through the Piston*
}

\author{
By Shoichi Furuhama**, Yositane Oya***, \\ and Tomikazu NAKAMURA****
}

On the assumption that the oil comes up by the action of "a non-return motion of the oil", the amount of oil which comes up through the clearances about the oil control ring and the top land of the piston was calculated on the basis of the hydrodynamical theory.

A Nissan's automotive engine ( $1200 \mathrm{cc}, 4$ cylinder water cooling) was run on the bench, and the oil loss when these clearances were changed was measured by a special precision device.

As a result, the following fact was obtained, i. e. the clearance between the cylinder wall and the top land of the piston, and the perfectness of the contact of the oil control ring with the upper side of the groove, and also with cylinder wall extremely affect the oil loss.

\section{Introduction}

The amount of oil loss from the piston skirt to the combustion chamber passing through the piston is one of the most important problems in the internal combustion engine, but at present, the greater part of this phenomenon remains still unsolved.

Hitherto some basic experiments(1)(2) were attempted to clear up its cause, and other measurement data(3)(4) obtained from actually working engines are available. But, we feel, the results of these studies are no more than preliminary data on the subject. We made an effort to obtain the basic mechanism of the motor oil loss, too.

In the first part of this work, it was supposed that the most dominant factor of this complex phenomenon was the hydrodynamic behavior of lubricating oil in the clearance among the cylinder, the piston and the piston ring, and the amounts of

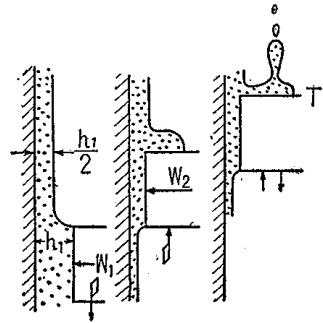

(i) Change of oil film

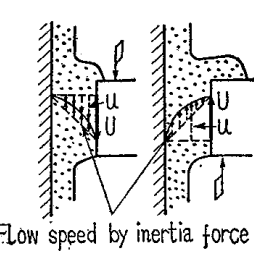

(ii) Inertia force

Fig. 1 Movement of return and non-return oil oil flow between these clearances are calculated theoretically.

In the second part, the oil losses from an actual automotive engine were measured by means of our new method.

\section{Imaginable movement of oil}

\subsection{Return flow and non-return flow}

The oil flow in a clearance between the cylinder and the piston would be easily determined by computation, if the clearance is filled with oil only without any amount of gas at all.

But in fact, the oil and gasses are coexistence, thereby the problem is made much more difficult.

The idea of the non-return flow is shown in Fig. 1 , and in the case (i) an oil film thickness in the upward travel is thinner than in the downward one.

Case (ii). Oil is collected on the edge of the piston or the ring by inertia force.

In these cases, any excess oil is scraped up necessarily, and one part of this oil which exists just near the entrance of the clear. ance can return again in the successive stroke that permits a thick oil film. But the other part of oil cannot be returned and lost in the combustion chamber.

Thereupon in the case of a tapered ring, for example, as showing in Fig. 1 (iii), the scraped oil is held in an easy returnable position, so the amount of oil up is small.

This theory on the effect of the

* Received 30th November, 1961.

* Professor, Musashi Institute of Technology, Setagaya-ku, Tokyo.

*** Chief Engineer, Nissan Motors Co., Ltd.

**** Lecturer, Musashi Institute of Technology. (iii) Tapered surface serves to return oil taper ring is different from Mr. Dykes's ${ }^{(2)}$ opinion.

Since the ratio of the non-return to the returnable oil has not yet been determined.

In this report, the calculation goes no further 
than to cover the amount of oil to be scraped up which will provide the basis to determine the nonreturn one.

\subsection{Amount of oil to be scraped up by piston} rings

We divide an oil flow into the three parts as Fig. 2: (i) a flow out from the top of piston I-I, (ii) scraped up by the piston rings, (iii) oil supply into the ring belt from II-II.

According to the working conditions of the experimental engine and using the Furuhama's theory ${ }^{(5)(6)(7)}$ on the piston-ring-lubrication, the minimum oil film thickness of each piston rings to the piston stroke are calculated.

Fig. 3 shows this result. This calculation is based on the assumption that each piston ring is fed with enough oil. However, when the oil film on the oil ring is thinner than on the compression ring that runs behind the oil ring, the oil to the compression ring is lacking, so a thinner oil film is inevitably formed. On the contrary, if either the oil ring has a thicker oil film, or the compression ring is supplied with much oil from other routes, then the significance of the oil ring is lost. Therefore the oil flow through the oil ring $q_{2}$ is a very important factor for the oil loss.

By folding back the development diagram Fig. 3 into the form of Fig. 4, we can see that the amount of surplus oil scraped up due to changes in oil film thickness in the inlet and exhaust stroke corresponds to the oblique-lined areas.

Fig. 5 shows the amount of scraped-up oil of each cylinder obtained by multiplying the above surplus by the number of revolution per minute (or

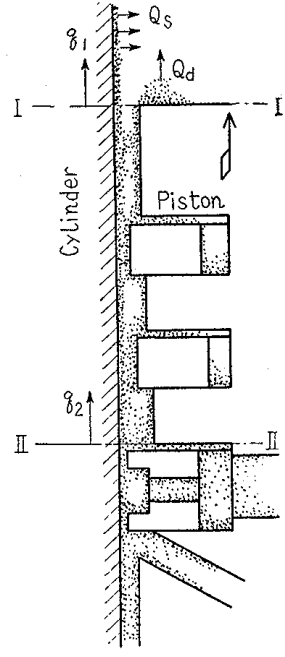

Fig. 2 Enlargement of the clearance around the piston rings

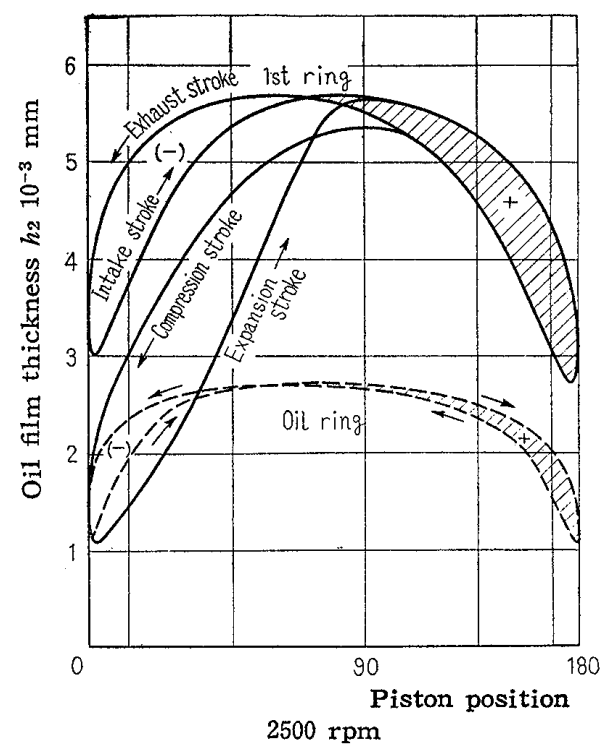

Fig. 4 Scraping up of oil due to change in oil film hitckness

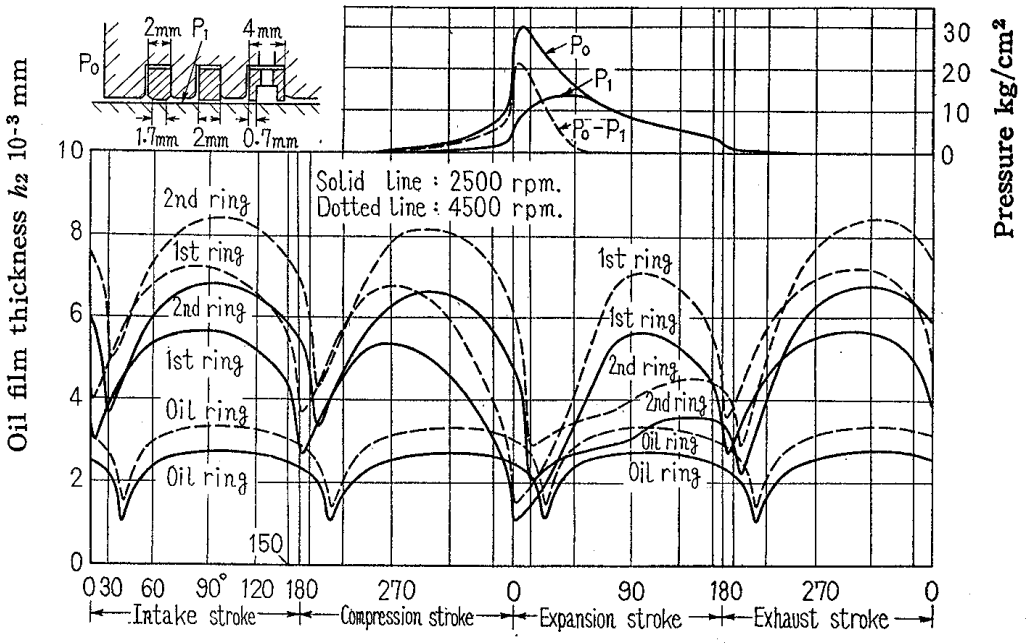

Lubricating oil : SAE-20

Piston position

Oil temperature : $100^{\circ} \mathrm{C}\left(\mu=7.2 \times 10^{-8} \mathrm{~kg} \mathrm{sec} / \mathrm{cm}\right)$

Fig. 3 Change of oil film thickness on the piston rings in ample oil supply

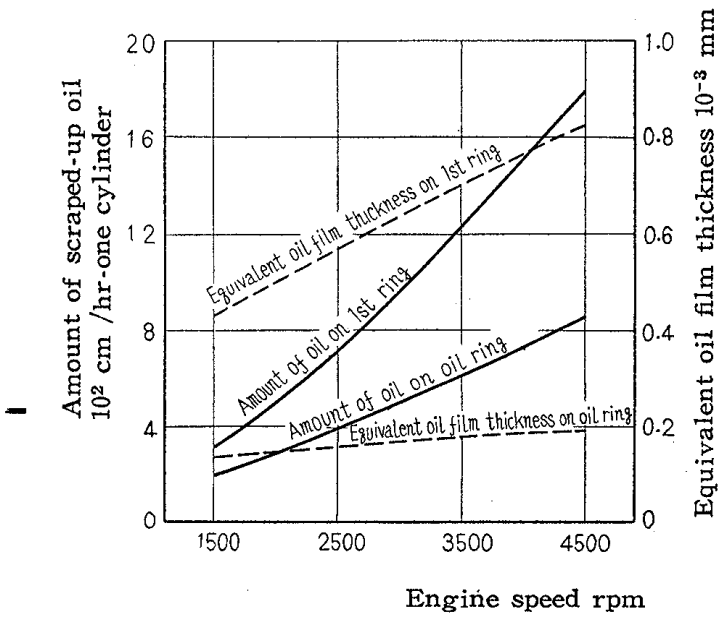

Fig. 5 Amount of oil scraped up withy changes in loil film thickness 
by $1 / 2$ of them for the 1st ring), and the average of oil film thickness determined by dividing it by the length of the stroke.

From these figures, we know that the amounts of oil to be scraped by the compression rings are very large and the amount of scraped up oil $(+)$ is nearly equal to the scraped down $(-)$. In other words, the piston ring has a large carrying ability of oil to upward or downward, that is, the oil reaches above the 1st ring as soon as it is supplied from the oil ring.

So in this report, the calculation is made on the flow rate of the cases (i) and (iii).

\section{Theoretical calculation}

\subsection{Oil flow on the top land due to inertia} force

Suppose as shown in Fig. 6 (a), that the piston top land is parallel to the cylinder wall and is filled with an oil of viscosity $\mu$, the piston moves at a speed $U_{0}$ and acceleration $\alpha_{0}$, while the cylinder remains still. Then the equilibrium equation of the forces acting at the points $x, y$ in the oil film, will be

$$
\frac{\partial^{2} u}{\partial y^{2}}=\frac{1}{\mu}\left\{\frac{\partial p}{\partial x}+\frac{\gamma}{g} \alpha\right\}
$$

where $\partial p / \partial x$ : pressure gradient, $\gamma:$ density of oil, $\alpha$ : acceleration, $u$ : speed at the point, $g:$ acceleration by specific gravity.

Now we supposed approximately

$$
\alpha=\alpha_{0}(h-y) / h
$$

Integrating Eq. (1) with respect to $y$ and applying the boundary conditions of $u=U_{0}$ at $y=0$ and $u=0$ at $y=h$, we obtained the velocity of oil at $y$.

$$
\begin{aligned}
u= & \frac{1}{2 \mu} \frac{\partial p}{\partial x}\left(y^{2}-h y\right)+\frac{\gamma \alpha_{0}}{6 g \mu h}\left(3 h y^{2}-y^{3}-2 h^{2} y\right) \\
& +U_{0}\left(1-\frac{y}{h}\right) \ldots \ldots \ldots \ldots \ldots \ldots \ldots \ldots \ldots \ldots \ldots \ldots \ldots \ldots \ldots \ldots \ldots \ldots \ldots
\end{aligned}
$$

Then the volume-continuity equation is

$$
\frac{\partial v}{\partial y}=-\frac{\partial u}{\partial x}
$$

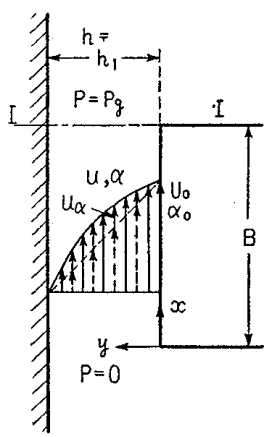

(a)

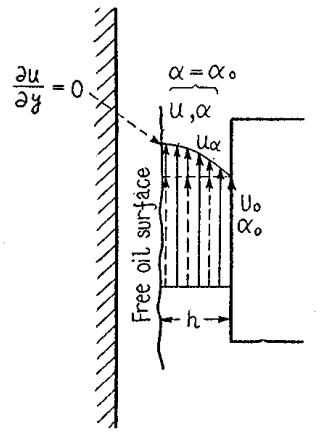

(b)
Fig. 6 where $v=0, p=0$ at $x=0$ and $p=P_{g}$ at $x=B$

$\frac{\partial p}{\partial x}=P_{g} / B$

If there is no pressure difference between the top and the low edge on the land, $\partial p / \partial x$ in Eq. (5) is zero, so the first term on the right side of Eq. (3) vanishes.

Therefore the oil flow rate per $1 \mathrm{~cm}$ of the circumferential length will be

$$
q=\int_{0}^{n} u d y=\frac{U_{0}}{2} h-\frac{\gamma \alpha_{0}}{24 g \mu} h^{3} \equiv q_{u}-q_{\alpha} \cdots \cdots \cdots
$$

where,

$$
\left.\begin{array}{l}
U_{0}=r w\left(\sin \omega t+\frac{1}{2 \lambda} \sin 2 \omega t\right) \\
\alpha_{0}=r w^{2}\left(\cos \omega t+\frac{1}{\lambda} \cos 2 \omega t\right)
\end{array}\right\}
$$

Amount of oil that flows in time $t$ from T.D.C. $(t=0)$

$$
\begin{aligned}
Q= & \int_{0}^{t} q d t=\frac{h}{2} r\left\{1-\cos \omega t+\frac{1}{4 \lambda}(1-\cos 2 \omega t)\right\} \\
& -\frac{r h^{3} r w}{24 g \mu}\left(\sin \omega t+\frac{1}{2 \lambda} \sin 2 \omega t\right) \equiv Q_{u}-Q_{\alpha} \cdots \text { ( 8) }
\end{aligned}
$$

where $Q_{u}$ is caused by the static motion, that is the velocity distribution is a straight line as indicated with the dotted line in Fig. 6, so it is zero unquestionably for one cycle.

Then $Q_{\alpha}$ is caused by inertia force, and the calculated value for one cycle is zero in the same way as $Q_{u}$; however, near the T.D.C. $Q_{\alpha}$ becomes the "non-return flow".

So during the upper half stroke

$Q_{T}=0.542 \frac{\gamma r h^{3} n^{2}}{g \mu} \mathrm{cc} / \mathrm{cm} \mathrm{hr}$



Fig. 7 Influence of clearance and $\mathrm{rpm}$ on the oil flow rate from the top land 
When the oil coating on the piston is kept off the cylinder wall as in Fig. 6 (b), on the assumption that (a) the oil at every position $x, y$ receives the equal inertia force caused by acceleration $\alpha_{0}$ and (b) $\partial u / \partial y=0$ on the free surface of $y=h$, we get

$$
q^{\prime}=U_{0} h-\frac{\gamma \alpha_{0}}{3 g \mu} h^{3}, \quad Q_{T^{\prime}}=4.33 \frac{\gamma r h^{3} n^{2}}{g \mu}
$$

The comparison of the above value $Q_{T}{ }^{\prime}$ with $Q_{T}$ in Eq. (9) shows that this flow rate equals that which arises from the double oil film thickness in the case (a).

In the case of the experimental engine, $r=3.55$ $\mathrm{cm}$ and $\gamma=0.88 \times 10^{-3} \mathrm{~kg} / \mathrm{cc}, \mathrm{Eq}$ (9) gives the results as shown in Figs. 7 and 8 , and these absolute values are far greater than the experimental values which are ascribed to the causes; (a) only a small portion of the whole circumference is filled up with oil film, (b) oil can flow into circumferential direction while the computation is based on two dimensions, and (c) there is a "returnable flow".

3.2 Amount of oil flow through the oil ring

By the law of continuity of flow at II-II in Fig. 9 the relationship of each flow is expressed as

$$
Q_{2}=\int_{T}^{B} q_{4} d t+\int_{T}^{B} q_{s} d t+2 r C_{g} h_{5}
$$

where $C_{g}$ is the size of the ring gap, so the third term on the right side is the amount of flow from this clearance, and the first one was treated in section $2 \cdot 2$.

Therefore we consider the flow of the second term. Generally, when a motion has a relative speed $U_{0}$, acceleration $\alpha_{0}$, and vertical speed $\partial h / \partial t$ between the parallel surfaces as illustrated in Fig.

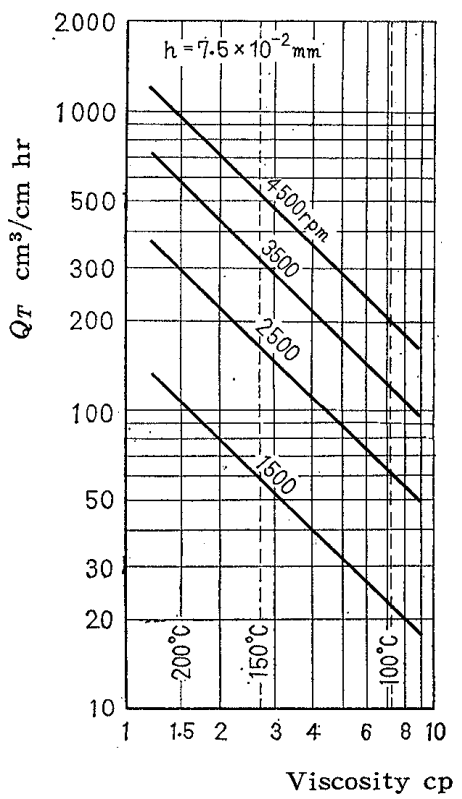

Fig. 8 Influence of Iviscosity on the oil flow rate from the top land
10, the oil flow at a point $x, y$ will be

$$
\begin{aligned}
q= & \frac{U_{0}}{2} h-\frac{\partial h}{\partial t}\left(x-\frac{L}{2}\right)-\frac{h^{3}}{12 \mu L}\left(P_{0}-P_{i}\right) \\
& -\frac{\gamma h^{3}}{24 g \mu} \alpha_{0} \ldots \ldots \ldots \ldots \ldots \ldots \ldots \ldots \ldots \ldots \ldots \ldots \ldots \ldots \ldots \ldots \ldots
\end{aligned}
$$

In the case of $q_{4}$ all other terms than the first are negligible, so

$$
q_{4}=U_{0} h_{4} / 2
$$

and for $q_{3}$, the second term becomes zero, and accordingly

$$
q_{3}=\frac{U_{0}}{2} h_{3}-\frac{1}{12 \mu} \frac{P_{A}-P_{2}}{l} h_{3}{ }^{3}-\frac{\gamma h_{3}{ }^{3}}{24 g \mu} \alpha_{0}
$$

For $q_{s}$ the first and fourth terms are zero (current is reverse), then:

$$
q_{s}=-\left(\frac{1}{12 \mu} \frac{P_{B}-P_{2}}{T} h_{s}{ }^{3}+\frac{T}{2} \frac{\partial h}{\partial t}\right)
$$

Substituting $P_{2}$ that is obtained from $q_{3}=q_{4}+q_{s}$ and $P_{A}=P_{B}=0$ into Eq. (15),

$$
\begin{aligned}
q_{s}= & \frac{h_{s}{ }^{3}}{T\left(\frac{h_{3}{ }^{3}}{l}+\frac{h_{s}{ }^{3}}{T}\right)}\left(\frac{U_{0}}{2} h_{3}+\frac{T}{2} \frac{\partial h}{\partial t}+\frac{\gamma h^{3}{ }^{3} \alpha_{0}}{24 g \mu}\right) \\
& -\frac{T}{2} \frac{\partial h}{\partial t} \ldots \ldots \ldots \ldots \ldots \ldots \ldots \ldots \ldots \ldots \ldots \ldots \ldots \ldots \ldots \ldots \ldots \ldots \ldots
\end{aligned}
$$

For one stroke,

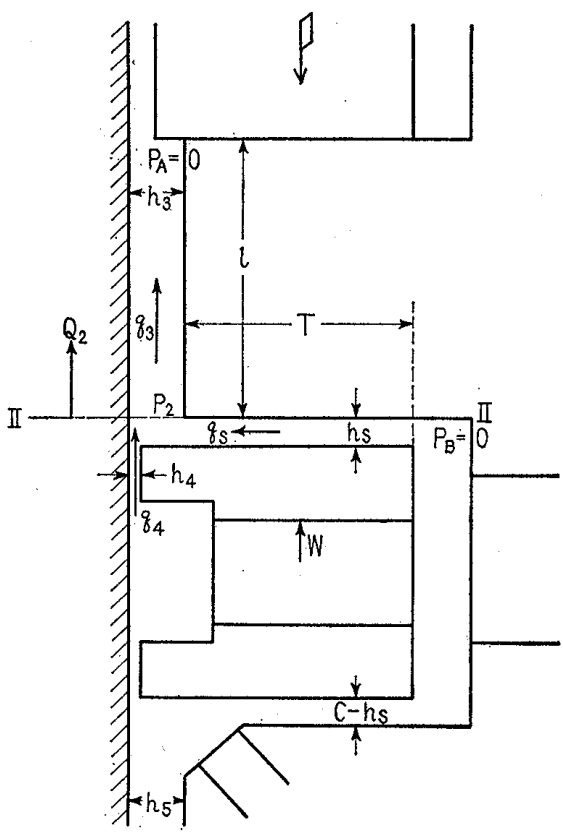

Fig. 9 Symbols of the clearance around the oil ring

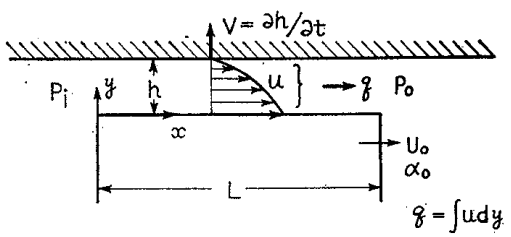

Fig. 10 Oil flow between the parallel surfaces 


$$
\begin{aligned}
Q_{\text {stroke }}= & \int_{T}^{B} \frac{h_{s}{ }^{3}}{T\left(\frac{h_{3}{ }^{3}}{l}+\frac{h_{s}{ }^{3}}{T}\right)}\left(\frac{h_{3}}{2} U_{0}+\frac{\gamma h_{3}{ }^{3}}{24 g \mu} \alpha_{0}\right) d t \\
& +\int_{T}^{B} \frac{h_{s}{ }^{3}}{2\left(\frac{h_{3}{ }^{3}}{l}+\frac{h_{s}{ }^{3}}{T}\right)} d h_{s}-\frac{T h_{s}}{2} \cdots \cdots . . .(17)
\end{aligned}
$$

In this equation, $\alpha_{0}$ of the second term in parenthesis of the first integration gives an equal value for the top and for the bottom dead centers, and the change of $h_{s}$ is symmetrical as calculated below.

Consequently this term becomes zero.

In the same way both the second integration and the last term, become zero.

Therefore

$Q_{\text {stroke }}=\int_{T}^{B} \frac{h_{3} h_{s}{ }^{3} U_{0}}{2 T\left(\frac{h_{8}{ }^{3}}{l}+\frac{h_{s}{ }^{3}}{T}\right)} d t$

Converting this value into the terms of each hour and each $1 \mathrm{~cm}$ of circumferential length:

$Q=60 n \cdot Q_{\text {stroke }}$

Integral of Eq. (18) must be computed by the numerical method.

Hence the main force acting on the oil ring vertically is the inertia force of itself, so $W$ in Fig. 11 becomes this inertia force.

And supposing that $W$ is supported by an oil pressure that is, by the squeeze velocity $-\partial h / \partial t$ on the surface of ring,

$$
\frac{\partial h}{\partial t}=\frac{\partial}{\partial x}\left(\frac{h^{3}}{12 \mu} \frac{\partial p}{\partial x}\right)
$$

Integrating this equation and from the boundary condition of $p=0$ when $x=0$ and $x=T$

$$
W=\int_{0}^{T} p d x=-\frac{\mu T^{3}}{h_{s}^{3}} \frac{\partial h}{\partial t}
$$

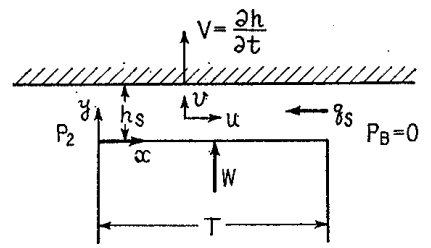

Fig. 11

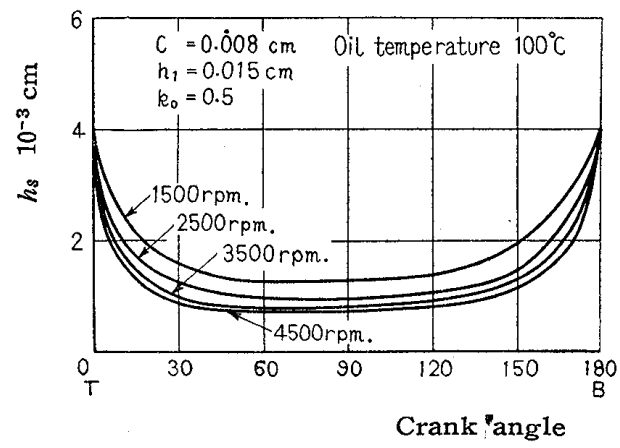

Fig. 12 Changes in $h_{s}$
On the under surface of the ring, similar resistance is acting against the clearance $C-h_{s}$.

Therefore,

$W=-\mu T^{3}\left\{\frac{1}{(C-h)^{3}}+\frac{1}{h^{3}}\right\} \frac{\partial h}{\partial t}$

Integrating,

$\int_{0}^{t} W d t=-\mu T^{3} \int_{0}^{l}\left\{\frac{1}{(C-h)^{3}}+\frac{1}{h^{3}}\right\} d h$

where,

$W=\frac{m}{g} r \omega^{2}\left(\cos \omega t+\frac{1}{\lambda} \cos 2 \omega t\right)$

$m$ is weight per $1 \mathrm{~cm}$ of the ring, $h_{s}=C k$ and $k$ is $k_{0}$ at $t=0$

Integrating,

$$
\begin{gathered}
\frac{\mu T^{3}}{2}\left\{\frac{1-2 k}{C^{2} k^{2}(1-2 k)^{2}}-\frac{1-2 k}{C^{2} k_{0}^{2}\left(1-k_{0}\right)^{2}}\right\} \\
=\frac{m}{g} \omega r\left(\sin \omega t+\frac{1}{2 \lambda} \sin 2 \omega t\right) \ldots .
\end{gathered}
$$

Now $k$ and $k_{s}$ can be evaluated by a graphical method, for example, in the case of the dimensions of our experimental engine, it is as shown in Fig. 12.

Substituting this value for $h_{s}$ in Eq. (18) the amount of oil flow is obtained as shown in Figs. 13, 14 and 15 .

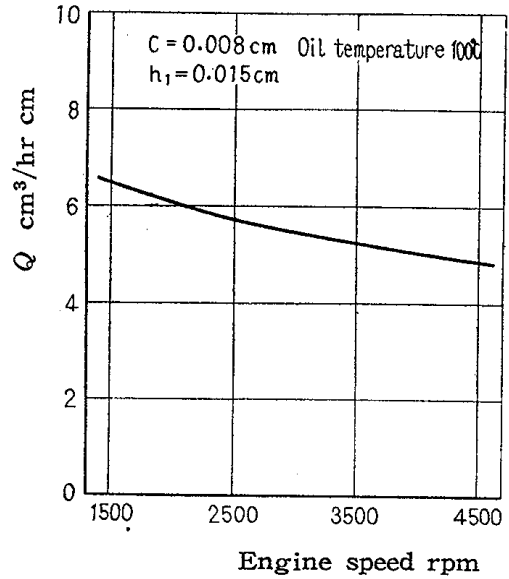

Fig. 13 Amount of up-flow of oil through $h_{3}$

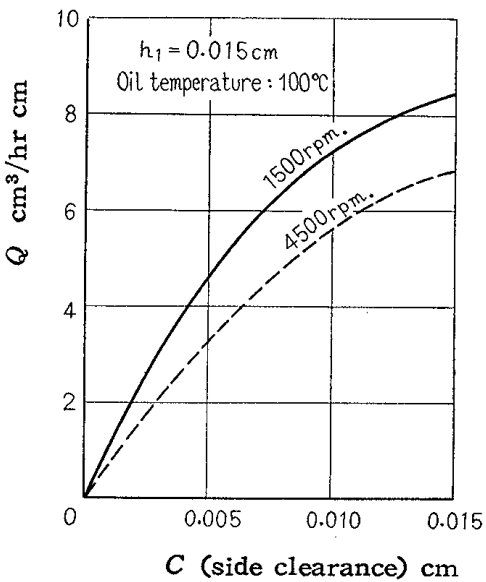

Fig. 14 Influence of $C$ on $q_{s}$ 
Now, when there is a constant value of $h_{s}$ due to the paor contact between the upper surface of the ring and the groove Eq. (18) becomes

$$
Q=60 n r \frac{h_{3} h_{s}{ }^{3}}{T\left(\frac{h_{3}{ }^{3}}{l}+\frac{h_{s}{ }^{3}}{T}\right)} \cdots \cdots \mathrm{cc} / \mathrm{cm} \mathrm{hr}
$$

It is shown that the influence of the constant clearance $h_{s}$ on the oil up is very important as in Fig. 16.

Finally the amount of oil film that remains constant on the skirt is given by

$$
Q=2 r C_{g} h_{5}
$$

where $r$ is half length of a stroke; $C_{g}$ : gap, $h_{5}$ : oil film thickness on the skirt=piston clearance.

As shown in Fig. 17 this value is unexpectedly very large.

\section{Experiment}

\subsection{Measuring device}

Lubricating oil consumption is usually measured by the methods: (a) changes in the total amount of oil in the sump and in circulation (measurement

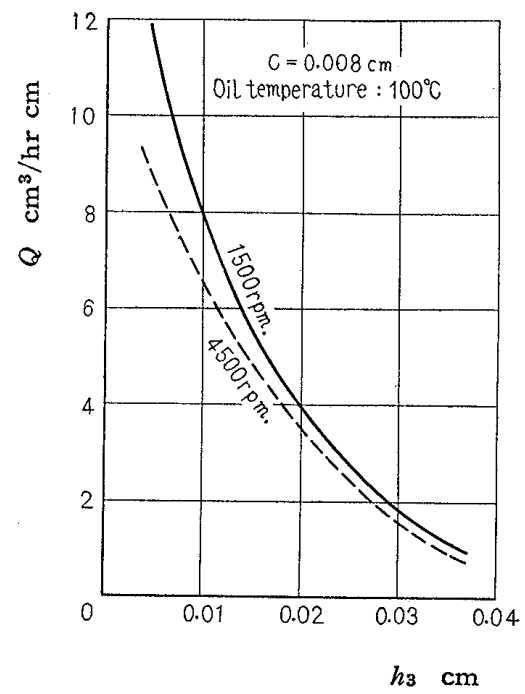

Fig. 15 Influence of $h_{3}$ on $q_{s}$

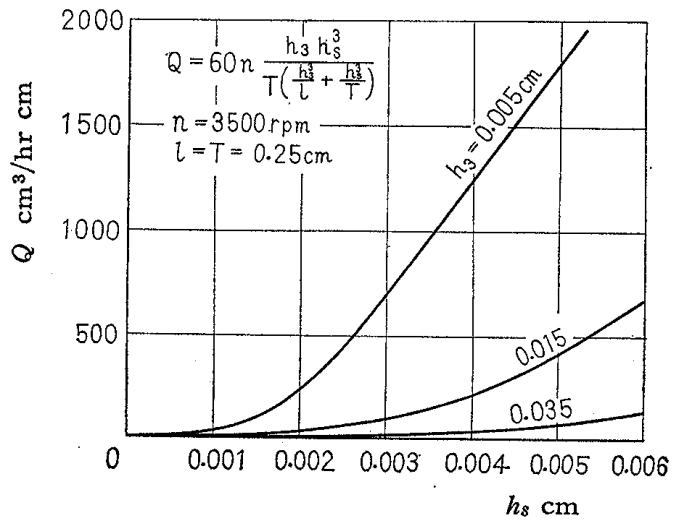

Fig. 16 Results of computation with $h_{s}$ constant of the engine overall weight), (b) exhaust gasses analysis (colour of exhaust gas, adherence of oil to the exhaust pipe, isotope detection, etc.) and (c) measuring of the amount of oil in the sump (as checked by weight or level).

In our study, the method (c) is also adopted.

Precisely speaking, however, we measured the oil pressure rather than the oil level, because in an actual working engine, the oil surface is rippled and contains small air bubbles.

In our measuring device, a float chamber is installed at a position where the engine motion is insulated, and the bottom of this chamber is connected with the engine sump through a large pipe.

The precise position of the float can be measured by means of such a device in which the electric indicator is deflected by the very small voltage when the upper part of float touches the bottom end of a micrometer.

In addition, an electric heater was provided in order to heat the chamber and connecting pipe at a constant temperature, so as to keep the oil at suitable viscosity.

Fig. 18 is a sectional view of the float chamber. Oil has free passage from the engine sump to the float chamber (2) through a pipe (1), while the space in the crankcase is connected with the upper space of the chamber.

The pipe (1) and the float chamber (2) are heated at a constant temperature of $50^{\circ} \mathrm{C}$ so that any change in the oil level in the engine sump may cause a ready response in the level to be measured.

As a contacting of the float tip (3) with the

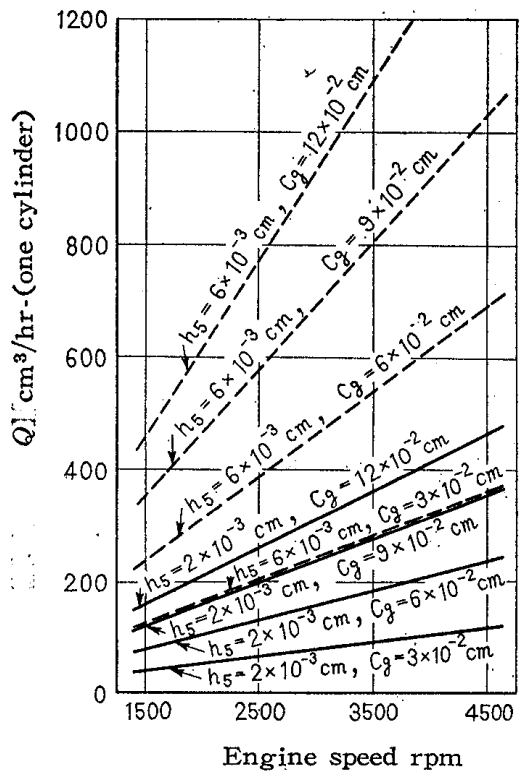

Fig. 17 Amount of oil flow passing the oil ring 
bottom end of the micrometer may cause an electric spark, which will not only damage them but will cause errors in measurement, the voltage of this current is reduced to any $M$. volts with $R$.

Using this device, we can obtain as high accuracy of oil level measurement as to several hundredths of a millimeter.

\subsection{Test engine and its operation}

For the experimental study, we use a Nissan Motors' $1200 \mathrm{cc}$, 4-cylinder, water cooled gasoline engine, with cylinder bore $73 \mathrm{~mm}$ and piston stroke $71 \mathrm{~mm}$, and details near the piston rings as shown in Fig. 19.

The engine lubrication system is such that oil is distributed by an oil pump from the sump to main bearings, the crank pins, cam shafts and

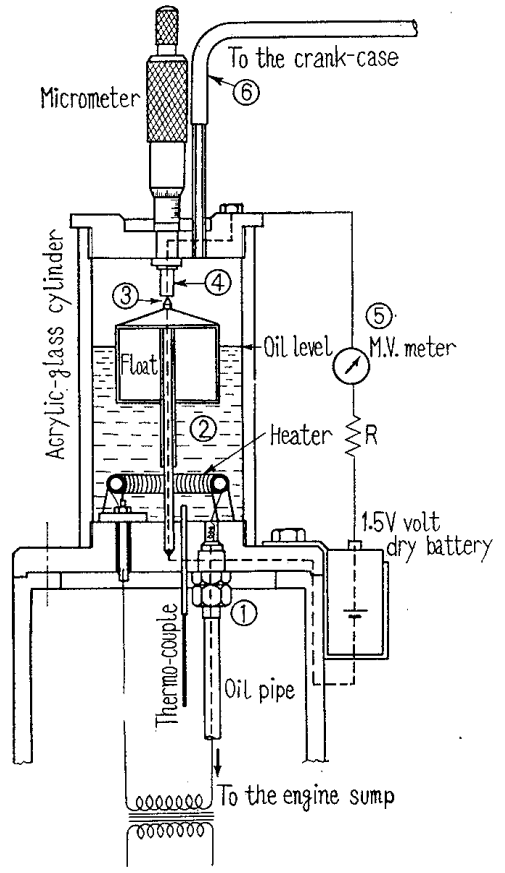

Fig. 18 Sectional view of the measuring device

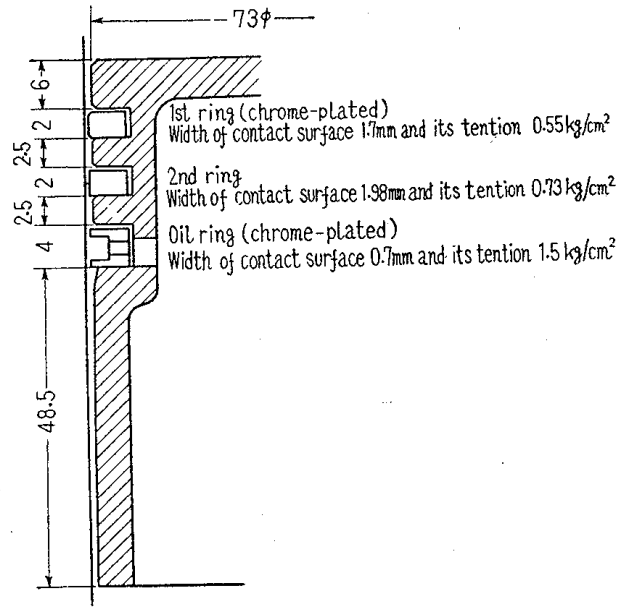

Fig. 19 Piston of the test engine over-head valves, through a filter, finally drifting back by gravity into the sump.

The piston-lubrication is forced-lubrication type where oil jets out into the bottom of the cylinder wall when a hole in the crank pin matches a hole in the connecting rod.

The change of the oil level in the sump is measured under a constant running for several hours under the given conditions mainly; with a half load by a Froud water dynamometer, the temperature of cooling water being controlled at $80^{\circ} \mathrm{C}$ at the outlet (and $65^{\circ} \mathrm{C}$ at the inlet), and the lubricating oil temperature is controlled by an oil cooler at $70^{\circ} \mathrm{C}$ at the inlet port of the oil pump.

\subsection{Results and considerations}

In the following data, some discrepancies are found between the absolute values of the tests carried out at long intervals of time between them.

However, we set all the conditions as identical as possible for each series of one experiment, so we can assure that the comparison values in one diagram are correct.

4.3.1 Influence of speed, load and temperatures

Fig. 20 shows the influence of the engine speed and oil temperature, and Fig. 21 the output.

Of these three factors the speed has the greatest influence, but the load and temperature

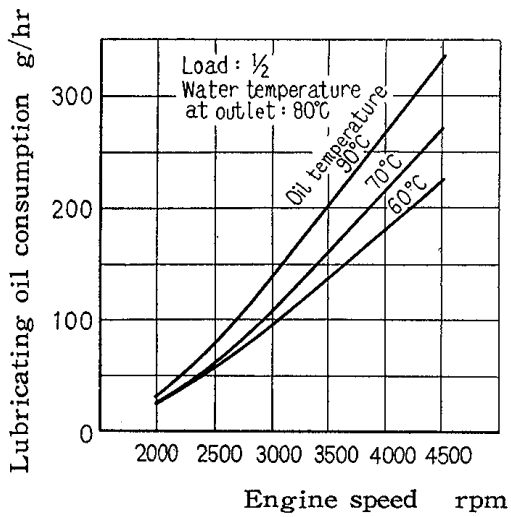

Fig. 20 Influence of revolution on the oil temperature

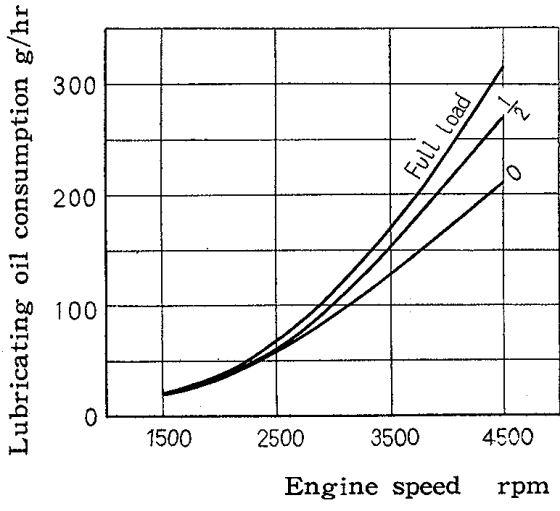

Fig. 21 Influence of load 
are much smaller than might be expected, and their influences are quite similar.

These results coincide well in tendency with those of the theoretical computation shown before in Figs. 7 and 8.

The characteristic of oil loss with the speed endorses the supposition that oil is lost mainly through the evaporation from the wall in the low speed range, while in the high speed range it is caused by inertia force.

4.3.2 Influence of the top land clearance $h_{1}$

Fig. 22 shows the results when the piston clearance of each land is changed. The fourth and fifth columns from the left cover the case where the clearance $h_{1}$ was set smaller, resulting in the most remarkable cut-down in the oil consumption.

From this it can be understood that the inertia force of oil on the top land has a great influence (see Fig. 7), and the clearance $h_{1}$ should be made

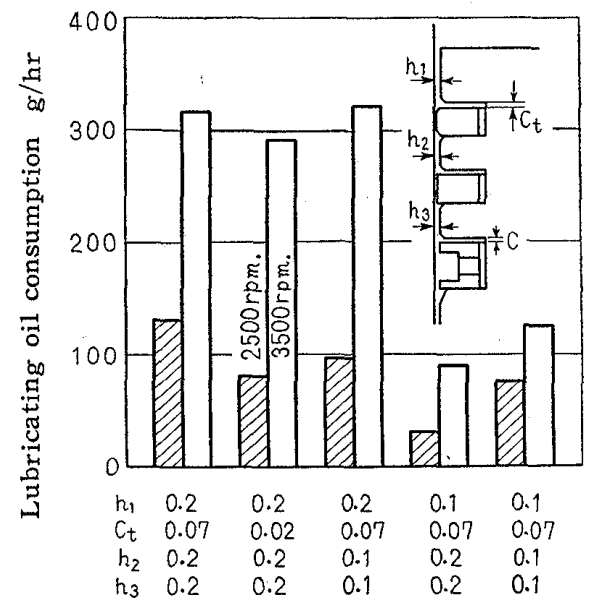

Fig. 22 Influence of the clearance around the top land

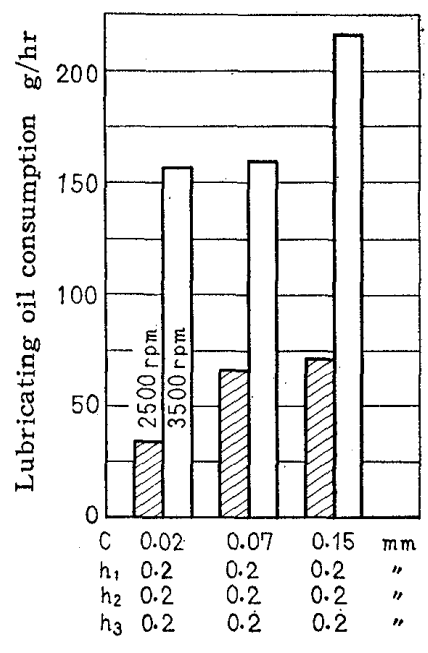

Fig. 23 Influence of the side clearance of the oil ring as small as possible.

Comparing the fifth ( $h_{2}$ and $h_{3}$ are small) with the fourth column, it is confirmed that the effect of $h_{3}$ agrees with the theoretical result.

4.3.3 Influence of the clearance around the oil ring Fig. 23 shows how much the oil loss increased when the side clearance on the oil ring was enlarged.

So far as the upper side of ring had a good contact this test brought about almost the same results as expected theoretically as shown in Fig. 14, that is to say, an increase in $C$ caused $a$ to rise in the oil loss slightly.

Fig. 24 shows the amount of oil loss when enlarging the clearance $h_{3}$ from $0.1 \mathrm{~mm}$ to $0.4 \mathrm{~mm}$ in cold, which corresponds to the theoretical calculation as shown in Fig. 15.

An increase from $0.1 \mathrm{~mm}$ to $0.2 \mathrm{~mm}$ did not bring about any remarkable change but when enlarged to $0.4 \mathrm{~mm}$, the oil loss decreased considerably.

This is a flow to be caused by negative pressure $P_{2}$.

Next the enlargement of the skirt clearance $h_{5}$ is considered to produce two effects, one is to increase the oil supply to the oil ring, and the other, to increase the amount of flow-out oil from the gap (see Fig. 17).

Our experiment is conducted further using the oil rings that have particular passage clearances of oil.

The results are illustrated in Fig. 25.

The third column from the left is the case of a ring which has six cuts of $1 / 100 \mathrm{~mm}$ deep on the contact surface with the cylinder as shown in the upper picture. This passage increases the oil loss very largely.

When the gap is enlarged from normal size $0.3 \mathrm{~mm}$ to $1 \mathrm{~mm}$, the oil loss rises as might be

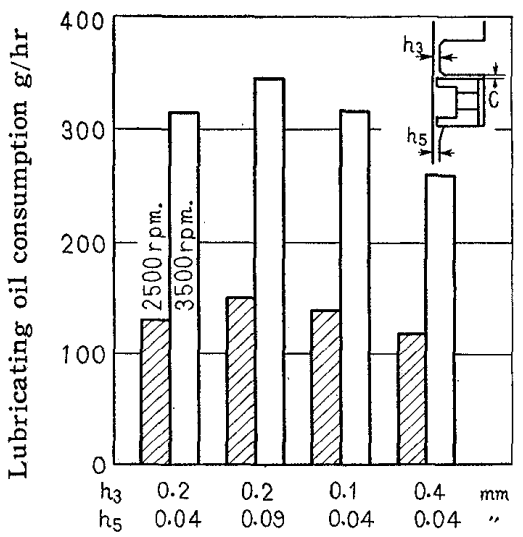

Fig. 24 Influence of $h_{3}$ 
expected theoretically in Fig. 17.

It is considered that the effect of enlarged gap and cut of outer periphery on the oil loss are due to the same route of oil flow.

Lastly the most important result is shown in the second column, where a ring has seven passages on the upper surface as shown in the under picture.

The most violent increase of oil loss is caused. But when the same ring turns upside down, so that the grooves may be down, the influence of the grooves is only negligibly small, this fact can be taken as corroborating the theoretical result as in Fig. 16.

Also these test results tell that the oil ring performs the function to stop the inflow of oil to the upper clearance like a check valve rather than to scrape down the oil.

\section{Conclusions}

(i) From the theoretical analysis and experimental results, we can understand the mechanism of the so-called oil-up as follows:

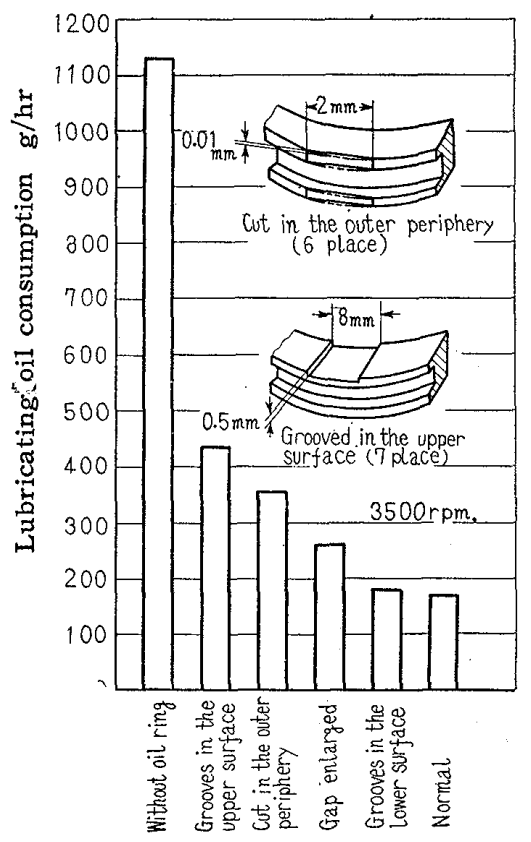

Fig. 25
Since the oil film on the oil ring is thinner than those on the other rings, the compression rings have always a lack of oil.

On the other hand, the compression ring has a characteristics to scrape a large amount of oil according to changes in the oil film thickness, therefore when oil is fed from the clearances of the oil ring, that is, the gap, clearance of upper surface and the cylinder, etc., this oil thickens the oil film of the compression rings and also it is brought up directly above the first ring.

Being sent there, it is energized by inertia force enough to pass the top land to the combustion chamber, where it either evaporates or is lost, being thrown off in splashes.

In the high speed range, the major part of the oil brought there is lost apparently in the latter way or is thrown off in splashes.

(ii) The amount of oil flow through a clearance under a certain pressure difference is in proportion to the third power of the size of the clearance. And the inertia force is in proportion to the second power of the number of revolutions.

From these facts, we can draw the conclusions: (a) the amount of oil loss increases particularly in the high speed range, (b) the effect of the top land clearance on oil loss by the inertia force is very large, and (c) the perfectness of the contact of the oil ring with the surfaces of the cylinder wall and the upper surface of groove has a close relation to the oil loss.

\section{References}

(1) S.J. Beaubien and A.G. Cattaneo:SAE Jour., Vol. 54, No. 10 (1946), p. 60.

(2) P. de K. Dykes : Proc, Inst. Mech. Engr., Vol, 171, No. 11 (1957), p. 413

(3) J.S. Coon and D.E. Loeffler: SAE Trans., Vol. 67, (1959), p. 59.

(4) G. WeiBbrod, H.G. Wenderoth, u. U.H. Chr. Wolff : $A T Z, \mathrm{Bd} .62, \mathrm{Nr} .11(1960), \mathrm{S}, 292$.

(5) S. Furuhama:Jour. Japan Soc. Mech. Engrs,, Vol. 64, No. 515 (1961), p. 1695.

(6) S. Furuhama : Trans. Japan Soc. Mech. Engrs., Vol. 24, No. 148 (1958), p. 1032.

(7) S. Furuhama: Trans, Japan Soc. Mech. Engrs., Vol. 27, No. 178 (1961), p. 919.

\section{Discussion}

T. MAsuba: (1) This paper gives valuable information on the amount of oil that will be consumed in the combustion chamber, depending upon the amount pumped into it by the piston and rings. Especially gratifying to me is the fact that he is trying to find good agreement between theory and experiment.
It is generally known that the rate of oil consumption is affected extremely by the engine revolution. In this paper, this problem is discussed by the author. Namely he says, in proportion as the engine revolution increases, the oil consumption of engine is increased because of the increased vaporized oil and the increased oil splashing by 


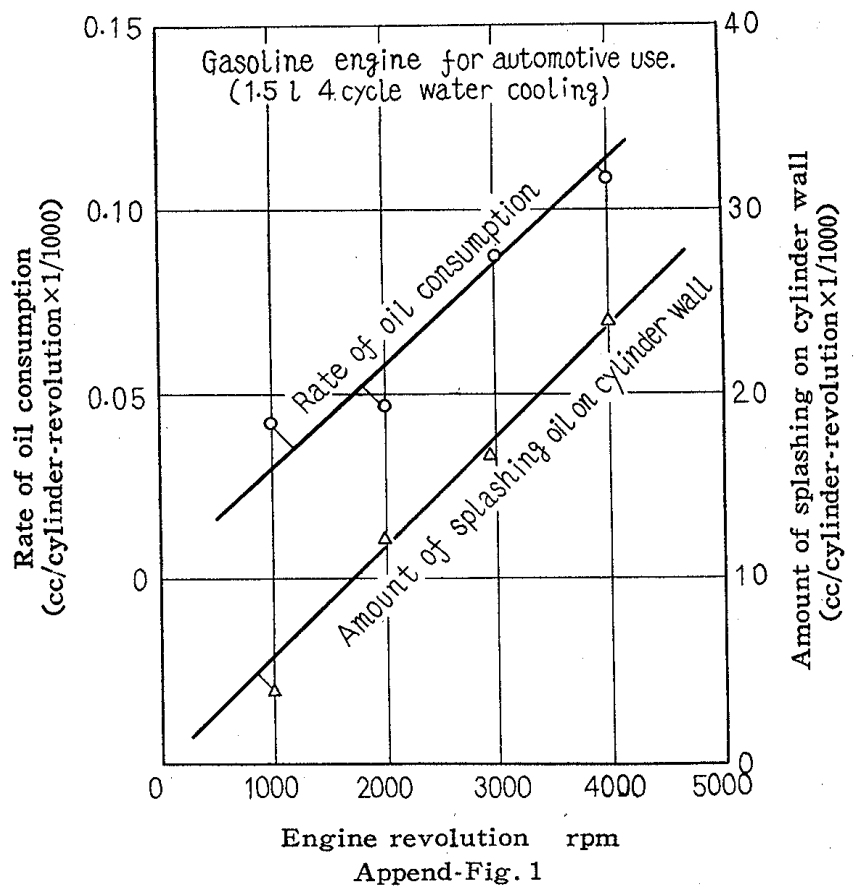

the inertia force of rings. However, I think the amount of splashing oil (on the cylinder walls) being thrown-off from the crankpin is a major factor in oil consumption. Because, as the engine revolution becomes higher, the amount of splashing oil on the cylinder walls is increased by the centrifugal force.

Append-Fig. 1 is an experimental example of oil consumption in our automobile gasoline engine. The feature of oil consumption is similar to that of the splashing oil on the cylinder wall.

K. SHImIz : (2) The discussor sincerely appreciates the efforts of the author in clarifying the influence of clearances in the cylinder on the "oil up" by uing theoretical formulas. As a matter of fact, it is difficult to plug up these clearances perfectly.

The "oil up" is generally believed to be caused by a vacuum formed at the top land of piston in the suction stroke and the discussor concurs perfectly with the author in the view that $h_{s}, C-h_{s}$ have hereby important bearing. The discussor wishes in this connection to suggest for reference that there is a special ring available which shows considerable effect for plugging the clearances; this ring is provided with side-rails of $0.6 \mathrm{~mm}$ high carbon steel in the expander portion.

\section{Authors' closure}

(1) As you suggest in the later tests we obtained the data showing that the oil loss was influenced largely by the amount of splashing oil.

It may be one of the sources that change the data incomprehensibly.

But yet we have not obtained the definite results on this phenomenon, so it will be a theme of future our study.

We believe that the results of your investigation are very significant.

(2) "A vacuum action of the suction stroke is a main factor of the oil-up" is also suggested by other investigators. But we cannot believe this theory because:

(i) The pressure difference between the crank case and the combustion chamber is decreased with piston rings step by step.

(ii) According to this theory of the pressure difference, more oil around the piston must flow down in the next compression and working stroke.

(3) As in your opinion, it is ascertained in our another experiment that the oil loss is made to decrease extremely by the expander ring pushing its sliding and side surface. It is supposed that the phenomenon is due to the limiting of $q_{2}$ in this report. But for the above reason, we are concerned about the wear resistance when the restriction of oil supply to the region of the compression ring is enforced so far. Thus this phenomenon will be a theme in our future studies. It will be very good if the oil can be supplied enough to the regions of the compression rings and more over the oil loss in the round of the top land can be prevented. But as you say, in practice there are many difficulties and we suppose it is necessary to study on the oil movements about the top land and the oil ring for the solution of this problem. 